\title{
Association between maternal dietary quality and gestational diabetes mellitus based on the Diet Balance Index for Pregnancy
}

\section{Shanshan Wang}

Huazhong University of Science and Technology Tongji Medical College School of Public Health Ning Wang

Huazhong University of Science and Technology Tongji Medical College School of Public Health Can Luo

Huazhong University of Science and Technology Tongji Medical College School of Public Health

\section{Rui Zhao}

Huazhong University of Science and Technology Tongji Medical College School of Public Health

\section{Yatan Du}

Huazhong University of Science and Technology Tongji Medical College School of Public Health

\section{Huihui Xie}

Huazhong University of Science and Technology Tongji Medical College School of Public Health

\section{Yuxuan Lu}

Huazhong University of Science and Technology Tongji Medical College School of Public Health

\section{Yue Xu}

Huazhong University of Science and Technology Tongji Medical College School of Public Health

\section{Dongxia Wang}

Huazhong University of Science and Technology Tongji Medical College School of Public Health

\section{Yan Li}

Huazhong University of Science and Technology Tongji Medical College School of Public Health

\section{Xuefeng Yang}

Huazhong University of Science and Technology Tongji Medical College School of Public Health

\section{Guoping Xiong}

Central Hospital of Wuhan: The Central Hospital of Wuhan

\section{Liping Hao ( $\nabla$ haolp@mails.tjmu.edu.cn )}

Huazhong University of Science and Technology Tongji Medical College School of Public Health https://orcid.org/0000-0001-8844-0892

\section{Research}

Keywords: Diet Balance Index, Gestational diabetes mellitus, Diet quality, Dietary patterns 
Posted Date: April 14th, 2021

DOl: https://doi.org/10.21203/rs.3.rs-413666/v1

License: (c) (i) This work is licensed under a Creative Commons Attribution 4.0 International License. Read Full License 


\section{Abstract}

Background: Maternal diet is critical to the development of gestational diabetes mellitus (GDM), but sparse studies have applied the Chinese Dietary Balance Index for Pregnancy (DBI-P) to assess the maternal dietary quality and its relationship with GDM. We aimed to examine the maternal dietary quality and its relationship with GDM risk using the newly developed DBI-P.

Methods: We included 1122 pregnant women from the Tongji Birth Cohort (TJBC) in Wuhan, China. The semi-quantitative food frequency questionnaire (FFQ) was used to obtain the dietary information during pregnancy. The newly developed DBI-P, including DBI-P components and DBI-P dietary patterns, was applied to comprehensively evaluate the maternal dietary quality. GDM was diagnosed by the 75-g, 2-h oral glucose tolerance test at 24-28 weeks gestation. We used a generalized linear regression model to examine the relationship between DBI-P components and blood glucose levels, and a logistic regression model to examine the relationship between dietary patterns and GDM.

Results: A total of 179 participants (16.0\%) were diagnosed with GDM. There is a widespread phenomenon of dietary imbalance among pregnant women in Wuhan. With per score increase in nuts and eggs, fasting blood glucose increase by $0.03 \mathrm{mmol} / \mathrm{l}(95 \% \mathrm{Cl}: 0.01,0.05)$ and $0.01 \mathrm{mmol} / \mathrm{l}(95 \% \mathrm{Cl}$ : $0.01,0.03)$, respectively, while per score increase in fruits, 1 -h post-load blood glucose decreased by 0.05 $\mathrm{mmol} / \mathrm{l}(95 \% \mathrm{Cl}:-0.10,-0.01)$. Besides, compared with pattern $\mathrm{E}$ characterized by higher intake of total energy, total fat, fruits, and cooking oil, pattern B (OR: $0.51,95 \% \mathrm{Cl}: 0.26,0.99)$ and pattern C (OR: 0.27 , $95 \% \mathrm{Cl}: 0.09,0.81)$ were associated with a lower GDM risk. The associations between dietary patterns and GDM risk may be partly attributed to the intakes of dietary total fat, carbohydrate, cholesterol, eggs, and cooking oil.

Conclusions: Unfavorable dietary quality during pregnancy is associated with a higher risk of GDM. The newly established DBI-P can provide an easy-to-implement tool to assess maternal dietary quality. These findings will provide new insights for the exploration of preferable dietary evaluation methods and prevention and control of GDM.

\section{Full Text}

Due to technical limitations, full-text HTML conversion of this manuscript could not be completed. However, the manuscript can be downloaded and accessed as a PDF.

\section{Figures}




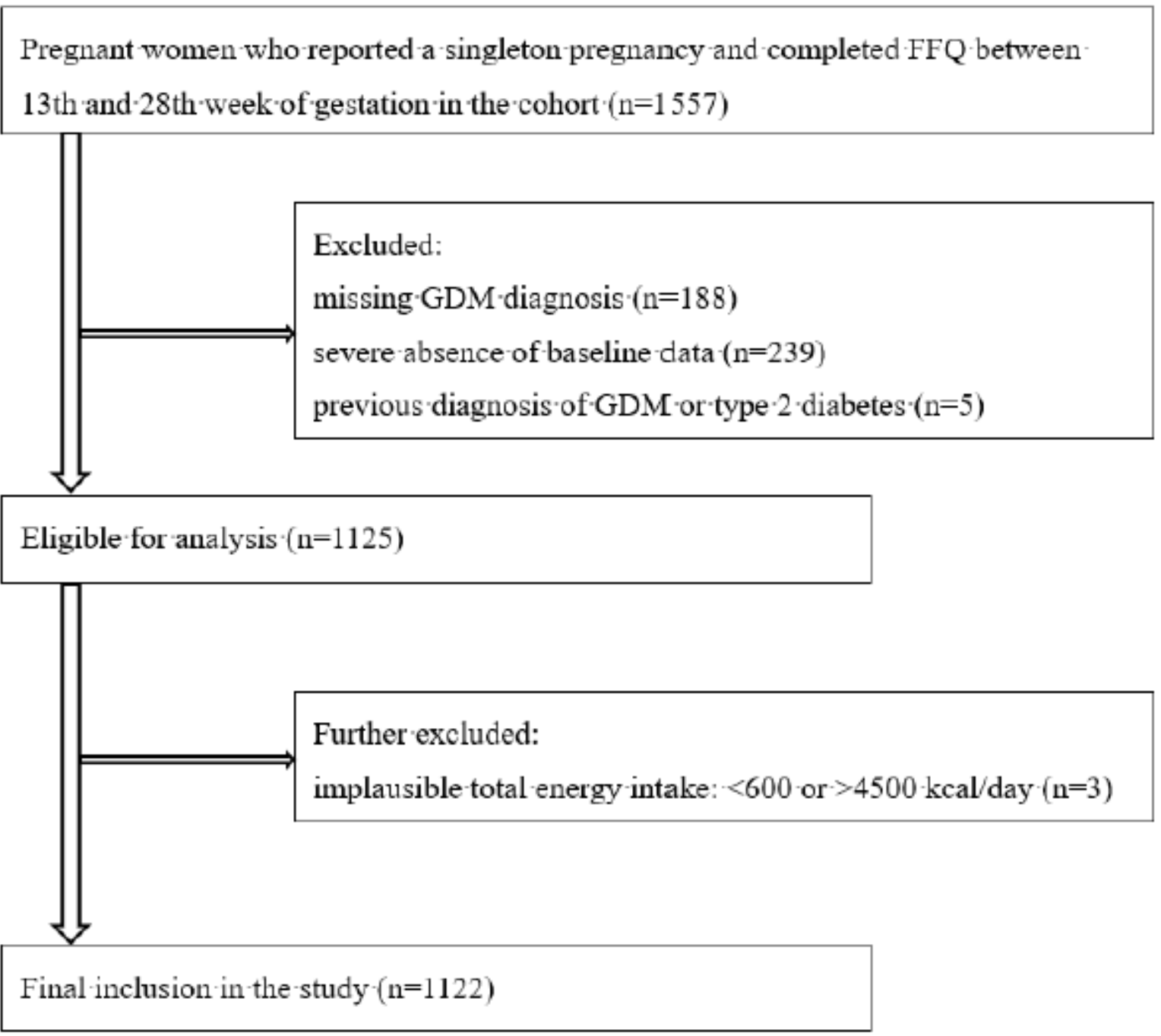

Figure 1

Flow chart for selection of study participants from TJBC study

\section{Supplementary Files}

This is a list of supplementary files associated with this preprint. Click to download.

- Additionalfile1.docx

- Additionalfile2.docx

- STROBEchecklistcohort.docx 